\title{
Analysis of Deformation Induced Grain Growth and Texture Development in electrodeposited Nickel- a Quantitative Comparison between ACOM-STEM and in-situ X-ray Diffraction
}

Christian Kübel ${ }^{1,2}$, Aaron Kobler ${ }^{1,3}$, P.A. Gruber ${ }^{4}$, J. Lohmiller ${ }^{4}$, O. Kraft ${ }^{4}$, C. Braun ${ }^{5}$, M. Grewer ${ }^{5}$, R. Birringer $^{5}$, H. Hahn ${ }^{1,3}$

1. Institute of Nanotechnology (INT), Karlsruhe Institute of Technology (KIT), 76344 Eggenstein-

Leopoldshafen, Germany

2. Karlsruhe NanoMicro Facility (KNMF), Karlsruhe Institute of Technology (KIT), 76344 EggensteinLeopoldshafen, Germany

3. Joint Research Laboratory Nanomaterials (KIT and TUD) at Technische Universität Darmstadt (TUD), Petersenstr. 32, 64287 Darmstadt, Germany

4. Institute for Applied Materials (IAM) Karlsruhe Institute of Technology, 76021 Karlsruhe, Germany

5. Universität des Saarlandes, Lehrstuhl für Experimentalphysik, Campus D2 2, 66041 Saarbrücken, Germany

Understanding the deformation mechanisms in nanocrystalline (nc) metals and alloys is crucial for improving their performance and stability as needed for technical applications. In-situ deformation studies using XRD have contributed significantly to our current understanding of nc metals and their deformation processes. However, it is difficult to analyze details of the local structure and processes based on these bulk measurements. The local structure is classically investigated using BF/DF-TEM and STEM imaging techniques, but varying contrast contributions due to local orientation, bending and defects make an accurate interpretation difficult for truly nanocrystalline materials. Therefore, we are extending automated crystal orientation mapping (ACOM) into a quantitative tool for metallographic analysis of nanocrystalline metals, which can further be combined with 'fast' $\mu$ p-STEM imaging during in-situ deformation studies [1].

This presentation will focus on the quantitative analysis of the structure of electrodeposited nickel (nc $\mathrm{Ni}$ ) with a grain size of around $30 \mathrm{~nm}$, which has been deformed in uniaxial compression as well as in shear compression tests. Orientation maps (Fig. 1) of the initial and final state have been generated on a Tecnai F20 using the NanoMegas ASTAR ACOM system [2] and were further processed using a modified version of the MatLab based software Mtex [3]. Key points of these modifications for quantitative data analysis were 1) global optimization of the ACOM indexing parameters, 2) noise reduction and 3) filtering of the detected grains using the reliability and index quality of the ACOM analysis. With this approach typically around $90-95 \%$ of the area could be indexed well without filling gaps between grains. The resulting grain representation was quantitatively evaluated in terms of the orientation dependent grain size, twinning and the crystallographic texture. A comparison of these ACOM results with the global changes detected in-situ by synchrotron X-ray diffraction show an excellent agreement of both techniques for the grain size evolution and the texture development during deformation of nc Ni [Fig 2].

Ultimately, this analysis showed that the deformation behavior of nc Ni consists of a distinct sequence of elastic grain interaction, grain boundary sliding, grain rotation, dislocation activity and grain growth. The succession of the different deformation mechanisms leads to a specific texture observed both by XRD and ACOM [4]. 
References:

[1] A. Kobler, A. Kashiwar, H. Hahn, C. Kübel, Ultramicroscopy (2013) accepted.

[2] E.F. Rauch, J. Portillo, S. Nicolopoulos, D. Bultreys, S. Rouvimov and P. Moeck, Zeitschrift für Kristallographie, 225 (2010) 103.

[3] Bachmann, F. et al., Solid State Phenomena, 160 (2010), 63.

[4] J. Lohmiller, M. Grewer, C. Braun, A. Kobler, C. Kübel, V. Honkimäki, O. Kraft, R. Birringer, P.A. Gruber, Acta Mat. (2013) submitted.

[5] Support by the Deutsche Forschungsgemeinschaft (FOR714) is gratefully acknowledged.
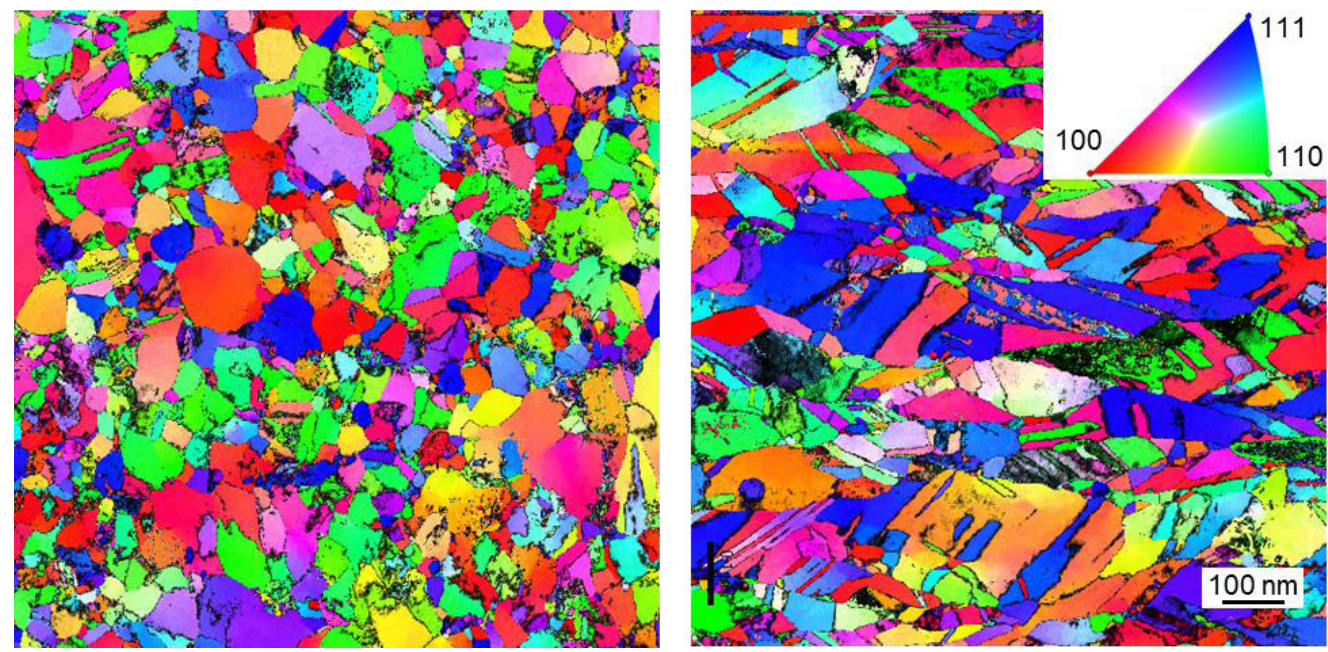

Figure 1. Plane-view and cross-section ACOM orientation maps of the electrodeposited nickel.

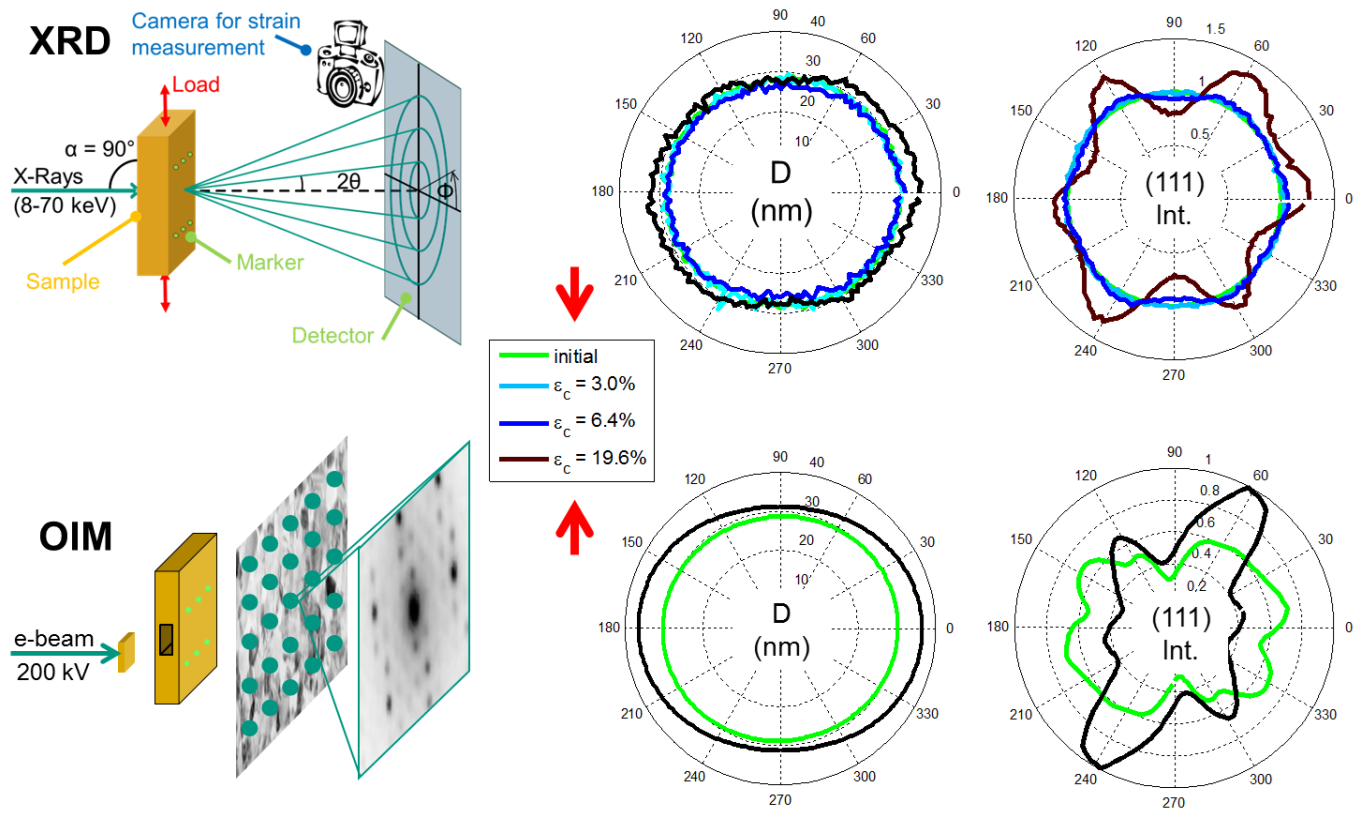

Figure 2. Quantitative analysis of the orientation dependent grain size and texture of the initial $\mathrm{nc} \mathrm{Ni}$ and after uniaxial compression reveals an excellent agreement between both global X-ray and local ACOM analysis. 